\title{
Subcortical Neural Synchrony and Absolute Thresholds Predict Frequency Discrimination Independently
}

\author{
F. Marmel, ${ }^{1}$ D. Linley, ${ }^{1}$ R. P. Carlyon, ${ }^{2}$ H. E. Gockel,${ }^{2}$ K. Hopkins, ${ }^{1}$ and C. J. Plack ${ }^{1}$ \\ ${ }^{1}$ School of Psychological Sciences, The University of Manchester, Manchester M13 9PL, UK \\ ${ }^{2}$ MRC Cognition and Brain Sciences Unit, Cambridge CB2 7EF, UK
}

Received: 3 September 2012; Accepted: 20 May 2013; Online publication: 13 June 2013

\begin{abstract}
The neural mechanisms of pitch coding have been debated for more than a century. The two main mechanisms are coding based on the profiles of neural firing rates across auditory nerve fibers with different characteristic frequencies (place-rate coding), and coding based on the phase-locked temporal pattern of neural firing (temporal coding). Phase locking precision can be partly assessed by recording the frequency-following response (FFR), a scalprecorded electrophysiological response that reflects synchronous activity in subcortical neurons. Although features of the FFR have been widely used as indices of pitch coding acuity, only a handful of studies have directly investigated the relation between the FFR and behavioral pitch judgments. Furthermore, the contribution of degraded neural synchrony (as indexed by the FFR) to the pitch perception impairments of older listeners and those with hearing loss is not well known. Here, the relation between the FFR and pure-tone frequency discrimination was investigated in listeners with a wide range of ages and absolute thresholds, to assess the respective contributions of subcortical neural synchrony and other age-related and hearing loss-related mechanisms to frequency discrimination performance. FFR measures of neural synchrony and absolute thresholds independently contributed to frequency discrimination performance. Age alone, i.e., once the effect of subcortical neural synchrony measures or absolute thresholds had been partialed out, did not contribute to frequency discrimination.
\end{abstract}

Correspondence to: F. Marmel - School of Psychological Sciences . The University of Manchester - Manchester M13 9PL, UK Telephone: +44-161-2756924; fax: +44-161-2753373; email: frederic.marmel@gmail.com
Overall, the results suggest that frequency discrimination of pure tones may depend both on phase locking precision and on separate mechanisms affected in hearing loss.

Keywords: FFR, sensorineural hearing loss, pitch perception, neural phase locking, age

\section{INTRODUCTION}

The neural mechanisms of pitch, the sensation whose variation is associated with melodies (Plack 2005), have been debated for more than a century (de Cheveigné 2005). Possible mechanisms rely on the profiles of neural firing rates across auditory nerve fibers with different characteristic frequencies (place-rate coding), and on the temporal firing patterns of auditory neurons that phase lock to sound periodicities (temporal coding). For low-frequency pure tones (up to $4-5 \mathrm{kHz}$ ), temporal mechanisms based on phase locking are thought to be dominant (Moore 1973a, b; Sek and Moore 1995; Micheyl et al. 1998; Heinz et al. 2001; Moore and Carlyon 2005). In particular, for listeners with a cochlear hearing loss (CHL), the generally observed degradation in frequency discrimination is not well explained in terms of frequency selectivity (Tyler et al. 1983; Moore and Peters 1992). Hence, impaired frequency difference limens (FDLs) in CHL listeners may be better accounted for in terms of reduced phase locking precision than in terms of rateplace code degradation.

Experimental support for a detrimental effect of cochlear damage on phase locking is equivocal. In animals, Harrison and Evans (1979) did not observe 
phase locking deterioration following drug-induced loss of outer hair cells in guinea pigs, but Woolf et al. (1981) did - in a similar experiment on chinchillas. More recently, studies have reported that noiseinduced hearing loss in cats (Miller et al. 1997) and chinchillas (Heinz et al. 2010) did not result in impaired phase locking in single auditory nerve fibers, although across-channel timing differences were affected in the study of Heinz et al. (2010). In humans, a degradation of phase locking has been associated with CHL listeners (Plyler and Krishnan 2001) when using the frequency-following response (FFR). The FFR is a scalp-recorded electrophysiological response originating from the brainstem (Moushegian et al. 1973; Worden and Marsh 1968) that reflects synchronous population-wide neuronal activity phase-locked to stimulus-related periodicities. Although not all spike periodicities appear in the FFR, it reflects some of the synchronous activity of the brainstem neurons phase-locked to the stimuli and to each other. Plyler and Krishnan (2001) found that listeners with elevated hearing thresholds had poorer FFR phase locking to the second formant transition of a speech sound.

The FFR has also been used to relate neural phase locking to frequency discrimination in normal-hearing listeners. Fundamental frequency difference limens (F0DLs) were shown to be negatively correlated to periodicity strength in the FFR in two studies using iterated rippled noise (IRN) that varied in pitch salience (Krishnan et al. 2010, 2012): F0DLs decreased and periodicity strength in the FFR increased concomitantly as the number of iterations in the IRN was increased. In another study, masking the distortion products of unresolved complex tones (thus reducing information on lower harmonics introduced by the auditory system) concomitantly increased FODLs and decreased periodicity strength in the FFR (Smalt et al. 2012). Also, training in a pitch discrimination task for complex tones with different pitch contours improved the representation of periodicity strength in the FFR (Carcagno and Plack 2011). However, no correlation between the FFR neural phase locking and frequency discrimination was found in a study using pure tones (Clinard et al. 2010). Finally, it is worth noting that although the FFR may represent pitch-bearing periodicity information, evidence suggests that it is not a direct representation of pitch (Gockel et al. 2011).

The aforementioned FFR studies relating neural phase locking to frequency discrimination focused on normal-hearing listeners: The current study investigated the relation between FFR neural phase locking and FDLs in listeners with normal as well as with elevated hearing thresholds. A common confound in studies comparing normal-hearing and hearing-impaired listeners is that the latter are often older than the former, and it is known that temporal processing and the FFR are adversely affected by age (Strouse et al. 1998; Clinard et al. 2010; Hopkins and Moore 2011; Vander Werff and Burns 2011; Konrad-Martin et al. 2012; Parbery-Clark et al. 2012; Anderson et al. 2012). The current study aimed to test a wide range of ages and absolute thresholds and disentangle their respective contribution to FDLs by using partial correlations.

\section{MATERIALS AND METHODS}

\section{Participants}

Twenty-seven participants (14 female) with a wide range of ages and audiometric thresholds were recruited (see Table 1). Participants' ages ranged from 22 to 77 years. Participants' audiometric thresholds for octave frequencies between 250 and $8,000 \mathrm{~Hz}$ ranged from being all below $0 \mathrm{~dB}$ HL with a mean of $-8 \mathrm{~dB}$ HL, to being all above $40 \mathrm{~dB}$ HL with a mean of 62 dB HL. Audiometric thresholds were measured either with standard audiometry (VIASYS GSI ARROW audiometer with Telephonics TDH-39P headphones) or, for four normal-hearing participants and one participant with a high-frequency loss, with a three-alternative forced choice (two-down one-up) procedure tracking the $70.7 \%$ point on the psychometric function. In the latter case, custom MATLAB software was used with Sennheiser HD 650 headphones. For participants with thresholds greater than $20 \mathrm{~dB} \mathrm{HL}$, hearing loss was confirmed to be of sensorineural origin. All the procedures of the study were approved locally by the School of Psychological Sciences Ethics Committee, University of Manchester, and nationally by the National Health Service North West 3 Research Ethics Committee.

\section{Behavioral procedure}

Frequency difference limens (FDLs) were measured for $660-\mathrm{Hz}$ pure tones. This frequency was chosen because the low-pass characteristic of the FFR made necessary the choice of a low common test frequency for behavioral and FFR measurements. Tones had a duration of $200 \mathrm{~ms}$, including 10-ms raised-cosine rise/fall times, and were presented monaurally at $80 \mathrm{~dB}$ SPL in the best ear (as determined by audiometric thresholds, nine left and 18 right). A background dichotic threshold-equalizing noise (TEN; see Moore et al. 2000) set to $40 \mathrm{~dB} / \mathrm{ERB}_{\mathrm{N}}$ was added, gated with the tone. A three-interval, threealternative forced choice task was used. The interstimulus interval was set to $500 \mathrm{~ms}$. Two intervals contained the standard $660-\mathrm{Hz}$ pure tone and one interval (chosen at random) contained a comparison 
TABLE 1

Age (years), ear tested, audiometric thresholds, and absolute threshold for a rectangular narrow-band noise centered on $660 \mathrm{~Hz}$, for the 27 participants

\begin{tabular}{|c|c|c|c|c|c|c|c|c|c|}
\hline \multirow[b]{3}{*}{ Participant } & \multirow[b]{3}{*}{ Age } & \multirow[b]{3}{*}{ Ear tested } & \multicolumn{6}{|c|}{ Audiometric threshold ( $d B H L)$} & \multirow{3}{*}{$\begin{array}{l}\text { Absolute threshold (dB SPL) } \\
660 \mathrm{~Hz}\end{array}$} \\
\hline & & & \multicolumn{6}{|c|}{ Frequency $(\mathrm{Hz})$} & \\
\hline & & & 250 & 500 & 1,000 & 2,000 & 4,000 & 8,000 & \\
\hline 1 & 56 & Left & 50 & 40 & 25 & 30 & 70 & 50 & 50 \\
\hline 2 & $29^{a}$ & Right & 1 & 0 & -10 & -4 & 1 & 13 & 13 \\
\hline 3 & 62 & Right & 35 & 35 & 40 & 45 & 40 & 15 & 36 \\
\hline 4 & 35 & Left & 10 & 5 & 10 & 15 & 20 & 0 & 7 \\
\hline 5 & 68 & Right & 35 & 35 & 35 & 45 & 65 & 80 & 33 \\
\hline 6 & 37 & Left & 70 & 65 & 60 & 60 & 45 & 35 & 66 \\
\hline 7 & 26 & Right & 15 & 5 & 5 & 10 & 20 & 5 & 19 \\
\hline 8 & 23 & Right & -5 & 0 & -5 & -10 & -10 & 5 & 12 \\
\hline 9 & 66 & Right & 20 & 10 & 20 & 30 & 65 & 80 & 19 \\
\hline 10 & $22^{\mathrm{a}}$ & Right & -1 & -2 & -10 & -10 & -11 & -15 & 13 \\
\hline 11 & 31 & Right & 45 & 60 & 55 & 60 & 55 & 75 & 64 \\
\hline 12 & 27 & Right & 0 & 5 & -5 & 10 & -10 & 15 & 16 \\
\hline 13 & 51 & Right & 5 & 5 & 5 & 5 & 10 & 25 & 20 \\
\hline 14 & 77 & Right & 45 & 40 & 60 & 70 & 80 & 75 & 64 \\
\hline 15 & 64 & Left & 50 & 35 & 30 & 25 & 60 & 50 & 47 \\
\hline 16 & $56^{\mathrm{a}}$ & Left & 8 & 8 & 2 & 4 & 3 & 23 & 15 \\
\hline 17 & 26 & Right & 30 & 25 & 40 & 45 & 45 & 45 & 41 \\
\hline 18 & 24 & Left & 5 & 0 & 0 & -10 & 0 & 0 & 12 \\
\hline 19 & $23^{a}$ & Right & -2 & -5 & -8 & -6 & 1 & 2 & 12 \\
\hline 20 & $71^{\mathrm{a}}$ & Right & 8 & 3 & 12 & 16 & 40 & 52 & 14 \\
\hline 21 & 55 & Right & 20 & 10 & 5 & 5 & 5 & 40 & 14 \\
\hline 22 & 31 & Left & 0 & 5 & 0 & 0 & 10 & 0 & 0 \\
\hline 23 & 35 & Right & 15 & 10 & 10 & 15 & 15 & 20 & 25 \\
\hline 24 & 26 & Left & 10 & 10 & 5 & -5 & -10 & 5 & 13 \\
\hline 25 & 61 & Right & 5 & 5 & -5 & 0 & -5 & 10 & 16 \\
\hline 26 & 55 & Right & 5 & 0 & 10 & 0 & 15 & 25 & 9 \\
\hline 27 & 58 & Left & 25 & 20 & 15 & 20 & 65 & 80 & 20 \\
\hline
\end{tabular}

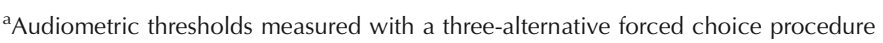

pure tone with a higher frequency. The listener was asked to detect the interval that was different from the two others by pressing a key on a computer keyboard. Visual feedback indicated whether the response was right or wrong. The percentage difference between the frequencies of standard and comparison was varied adaptively using a geometric track with a twodown, one-up rule. The maximum allowed difference was $200 \%$. If the track reached $200 \%$, incorrect responses did not result in an increase in the percentage difference. A block of trials consisted of 16 reversals (changes in track direction). The step size was a factor of 2 for the first four reversals and a factor of 1.414 for the remaining 12 reversals. For each block, the FDL was taken as the geometric mean of the frequency difference at the last 12 reversals. Each participant completed three blocks, and the geometric mean of the three FDLs was taken as the final estimate.

Absolute thresholds for a rectangular narrow-band noise centered on $660 \mathrm{~Hz}$ (200-ms duration including 10 -ms raised-cosine rise/fall times, noise width $=$
$132 \mathrm{~Hz}$, filtered in the frequency domain with infinite slopes) were measured for all participants using custom MATLAB software and a three-alternative forced choice procedure. The choice of a narrowband noise over a pure tone was to minimize the variability in threshold caused by individual differences in the location of peaks and valleys in the threshold microstructure (Cohen 1982).

\section{Electrophysiological procedure}

FFRs were recorded to pure tones at $660 \mathrm{~Hz}$ and neighboring frequencies $(620,640,664,680$, and $720 \mathrm{~Hz}$ ). Stimuli were $200 \mathrm{~ms}$ long, including 10-ms rise/fall times, and were generated in cosine phase at a $24,414 \mathrm{~Hz}$ sampling rate to match the sampling rate of the recording system used (TDT system 3 and BioSigRP software; Tucker-Davis Technologies, FL, USA). Using several frequencies allowed the derivation of a measure of FFR latencies without relying on onset responses-which were difficult to observe for pure tones-by calculating the group delay of the FFR 
responses, which is the slope of the linear variation of phase with frequency (Anderson et al. 1971; Batra et al. 1986). FFRs were recorded while participants reclined comfortably in an acoustically shielded booth. Stimuli were presented monaurally at $80 \mathrm{~dB}$ SPL in the best ear in quiet, at a rate of $3.18 \mathrm{~s}^{-1}$ using a mu-metal shielded ER-3A earphone (Etymotic Research, IL, USA). FFRs were recorded differentially between scalp electrodes placed on the ipsilateral mastoid (active) and the contralateral mastoid (reference). Another electrode placed on the forehead served as the common ground. The electrode impedances were maintained below $3 \mathrm{k} \Omega$. Recordings were bandpass-filtered online between $30 \mathrm{~Hz}$ and $3 \mathrm{kHz}$ and with a $50-\mathrm{Hz}$ notch bandstop filter. The acquisition rate was $24,414 \mathrm{~Hz}$.

FFR data were analyzed offline. About 2,300 stimulus presentations were averaged per frequency condition and participant (this value varied slightly due to differing numbers of sweeps being removed by the artifact rejection procedure). FFR measures included calculations of group delays, stimulus-to-response cross-correlations, and signal-to-noise ratios. First, group delays were calculated to estimate the onset delay of the FFR. For each participant and frequency condition, the phase of the averaged FFR at the signal frequency was computed with a discrete Fourier transform (without prior offline filtering or windowing). Unwrapped phases were then plotted against frequency and the slope of the regression line was taken as the group delay.

Second, cross-correlations between stimuli and responses in the time domain were calculated to estimate the responses' fidelity to the stimulus periodicity. The initial $20 \mathrm{~ms}$ of the responses (starting from the time when the stimulus starts playing) were discarded to restrict analyses to the most periodic part of the response (Lee et al. 2009; Marmel et al. 2011; Gockel et al. 2011), resulting in 180-ms long epochs. Crosscorrelations were performed (without prior offline filtering) over a range of time lags $(0$ to $15 \mathrm{~ms}$ ) by multiplying the waveforms of the stimuli and the FFR epochs (padded to $200 \mathrm{~ms}$ to match the stimuli length) and summing the results across time. Cross-correlations were normalized so that an autocorrelation at zero lag would be equal to 1 . The highest correlation factor across the range of time lags was taken as the crosscorrelation value.

Third, spectral signal-to-noise ratios (SNRs) were calculated to estimate the strength of the target frequency representation in the FFR. Again, the initial $20 \mathrm{~ms}$ of the responses were discarded. The FFRs were first filtered between 500 and 2,000 Hz (256th order FIR filter). Then power spectra were computed with a fast Fourier transform (FFT) using Hamming windowing and zero-padding to reach a $1-\mathrm{Hz}$ frequen- cy resolution. SNRs were calculated as ratios between the summed power magnitudes of a $21-\mathrm{Hz}$ frequency window centered on the signal frequency and the summed power magnitudes for the other frequencies between 500 and $1,000 \mathrm{~Hz}$, and then converted to $\mathrm{dB}$. For each of the two measures (cross-correlations and SNRs separately), measurements were averaged across the six frequency conditions to increase statistical power, resulting in measurements averaged over $\sim 13,600 \pm 10 \%$ sweeps per participant.

Finally, as statistical analyses showed very similar results for SNRs and cross-correlations ${ }^{1}$, results in the main text are reported for a composite FFR synchronization strength score obtained by calculating $\mathrm{z}^{-}$ scores separately for SNRs and cross-correlations and then averaging them. Composite FFR scores have been used previously to quantify pitch tracking (Russo et al. 2008), neural synchrony (Russo et al. 2009) and frequency encoding (Song et al. 2012). The composite FFR synchronization strength score in the present study reflects the fidelity of the FFR to the stimulus periodicity (higher scores reflect higher fidelity). The results for individual FFR measures (FFR cross-correlations and spectral SNRs) are also presented, in Table 2.

\section{Statistical analyses}

The mean FDLs were log-transformed in the statistical analyses to normalize their distribution. Pearson's correlations and partial correlations were computed with the following variables: age, absolute threshold for the narrow-band noise centered on $660 \mathrm{~Hz}, \log -$ transformed FDL, FFR group delay, spectral SNR, cross-correlation, and FFR synchronization strength. A linear regression analysis (stepwise method with backward selection) was also performed to determine which combination of the aforementioned variables best accounted for the FDLs. All statistical analyses were carried out with SPSS (SPSS, Chicago, IL, USA). Pearson's correlations and partial correlations are reported in Table 2 for all FFR measures of neural synchrony (spectral SNR, cross-correlation, and FFR synchronization strength). For the sake of brevity, the results section focuses on FFR synchronization strength, as the statistical analyses showed very similar

\footnotetext{
1 The use of only one FFR measurement, either the SNRs or the cross-correlations, instead of the composite measure of the two, does not change which correlations are significant and which are not, except in two cases: 1) the correlation between group delay and FFR strength misses significance when using cross-correlations $\left(r_{23}=\right.$ $-0.38, P=0.062$ ) (but it remains significant when using SNRs; $r_{23}=$ $-0.52, P=0.0078)$, and 2) the partial correlation between FFR and FDL with both age and absolute threshold partialed out misses significance when using SNRs $\left(r_{23}=-0.39, P=0.056\right)$ (but it remains significant when using cross-correlations; $\left.r_{23}=-0.40, P=0.046\right)$.
} 
TABLE 2

Summary table of Pearson's correlation coefficients and partial correlation coefficients between all variables defined in the 'Statistical Analyses' section: age, absolute threshold, FDL, FFR SNR, FFR cross-correlation, FFR synchronization strength (composite score obtained by calculating and averaging Z-scores for FFR SNR and FFR cross-correlation, see 'Materials and Methods'), and FFR group delay

\begin{tabular}{|c|c|c|c|c|c|c|}
\hline & Age & $\begin{array}{l}\text { Absolute } \\
\text { threshold }\end{array}$ & $F D L$ & FFR SNR & $\begin{array}{l}\text { FFE cross- } \\
\text { correlation }\end{array}$ & $\begin{array}{l}\text { FFR synchronization } \\
\text { strength }\end{array}$ \\
\hline Absolute threshold & 0.28 & & & & & \\
\hline FDL & $\begin{array}{l}0.39^{*} \\
\left(0.14^{\beta}\right)\left(0.15^{\gamma}\right)\end{array}$ & $\begin{array}{l}0.58^{* *} \\
\left(0.50^{\alpha \times \gamma}\right)^{*}\end{array}$ & & & & \\
\hline FFR SNR & $\begin{array}{l}-0.50^{* *} \\
\left(-0.46^{\beta}\right)^{*}\end{array}$ & -0.30 & $\begin{array}{l}-0.53^{* *} \\
\left(-0.39^{\alpha \times \beta}\right)\end{array}$ & & & \\
\hline FFR cross-correlation & $\begin{array}{l}-0.48^{*} \\
\left(-0.43^{\beta}\right)^{*}\end{array}$ & -0.32 & $\begin{array}{l}-0.55^{* *} \\
\left(-0.40^{\alpha \times \beta}\right)^{*}\end{array}$ & $\begin{array}{l}0.90^{* *} \\
\left(0.86^{\alpha \times \beta}\right)^{* *}\end{array}$ & & \\
\hline $\begin{array}{l}\text { FFR synchronization } \\
\text { strength }\end{array}$ & $\begin{array}{l}-0.51^{* *} \\
\left(-0.46^{\beta}\right)^{*}\end{array}$ & -0.32 & $\begin{array}{l}-0.56^{* *} \\
\left(-0.41^{\alpha \times \beta}\right)^{*}\end{array}$ & $\begin{array}{l}0.97^{* *} \\
\left(0.96^{\alpha \times \beta}\right)^{* *}\end{array}$ & $\begin{array}{l}0.97^{* *} \\
\left(0.96^{\alpha \times \beta}\right)^{* *}\end{array}$ & \\
\hline FFR group delay & $\begin{array}{l}0.52^{* *} \\
\left(0.42^{\beta}\right)^{*}\end{array}$ & $\begin{array}{l}0.47^{*} \\
\left(0.36^{\alpha}\right)\left(0.40^{\gamma}\right) \approx\end{array}$ & 0.31 & $\begin{array}{l}-0.52^{* *} \\
\left(-0.35^{\alpha}\right)\left(-0.46^{\beta}\right)^{*}\end{array}$ & -0.38 & $\begin{array}{l}-0.47^{*} \\
\left(-0.26^{\alpha}\right)\left(-0.40^{\beta}\right) \approx\end{array}$ \\
\hline
\end{tabular}

When correlations are significant, partial correlations are added below in brackets. The variables partialed out are labeled as follows: $\alpha$ for age, $\beta$ for absolute threshold, $\gamma$ for FFR synchronization strength, $\alpha \times \beta$ for both age and absolute threshold partialed out, and $\alpha \times \gamma$ for both age and FFR synchronization strength partialed out

$\approx p$ value between 0.05 and 0.06

${ }^{*} p<0.05 ;{ }^{* *} p<0.01$ (level of significance)

results when using SNR or cross-correlation (see Footnote 1 and Table 2).

\section{RESULTS}

\section{Subcortical origin of the electrophysiological responses}

The group delay for young normal-hearing participants was $6.5 \mathrm{~ms}$ on average. This rules out a cochlear microphonic origin of the responses (the latency of the cochlear microphonic was estimated to be $0.66 \mathrm{~ms}$ by Stillman et al. 1978) and is consistent with a subcortical origin of the responses.

FFR synchronization strength is related to pure tone frequency discrimination

The composite FFR synchronization strength scores were significantly correlated with the FDLs $\left(r_{25}=-0.56\right.$, $P=0.0025)$ : Stronger representations of the pure-tone frequency were associated with better (smaller) FDLs (Fig. 1A). The association between FFR synchronization strength and frequency discrimination could not be entirely attributed to a confounding effect of absolute threshold or age: FFR synchronization strength was significantly correlated with FDLs even when both absolute thresholds and ages were partialed out $\left(r_{23}=-0.41, P=0.042\right)$. However, the latencies of the FFR responses, as indexed by group delays, were not significantly correlated with FDLs $\left(r_{23}=0.31, P=0.13\right)$.

Absolute thresholds and FFR synchronization strength make independent contributions to pure tone frequency discrimination

Absolute thresholds were significantly correlated with FDLs $\left(r_{25}=0.58, P=0.0015\right)$ : Smaller absolute thresholds were associated with smaller (better) FDLs (Fig. 1B). The association between absolute thresholds and frequency discrimination could not be attributed to confounding effects of FFR synchronization strength or age: absolute thresholds were significantly correlated with FDLs even when both FFR synchronization strength and age were partialed out $\left(r_{23}=0.50, P=0.011\right)$. Absolute thresholds did not correlate significantly with FFR synchronization strength $\left(r_{25}=-0.32, \quad P=0.10\right)$ but they correlated significantly with group delays $\left(r_{23}=0.47, P=0.017\right)$, although not when age was partialed out $\left(r_{22}=0.36, P=0.088\right)$. These results, together with the relations between FFR synchronization strength and FDLs reported above, suggest independent contributions of absolute thresholds and FFR synchronization strength to frequency discrimination. More precisely, the results suggest that there are separate factors that influence absolute thresholds and FFR synchronization strength, and that these factors have independent effects on FDLs. For brevity, we use the term "independent contributions" throughout this 
A
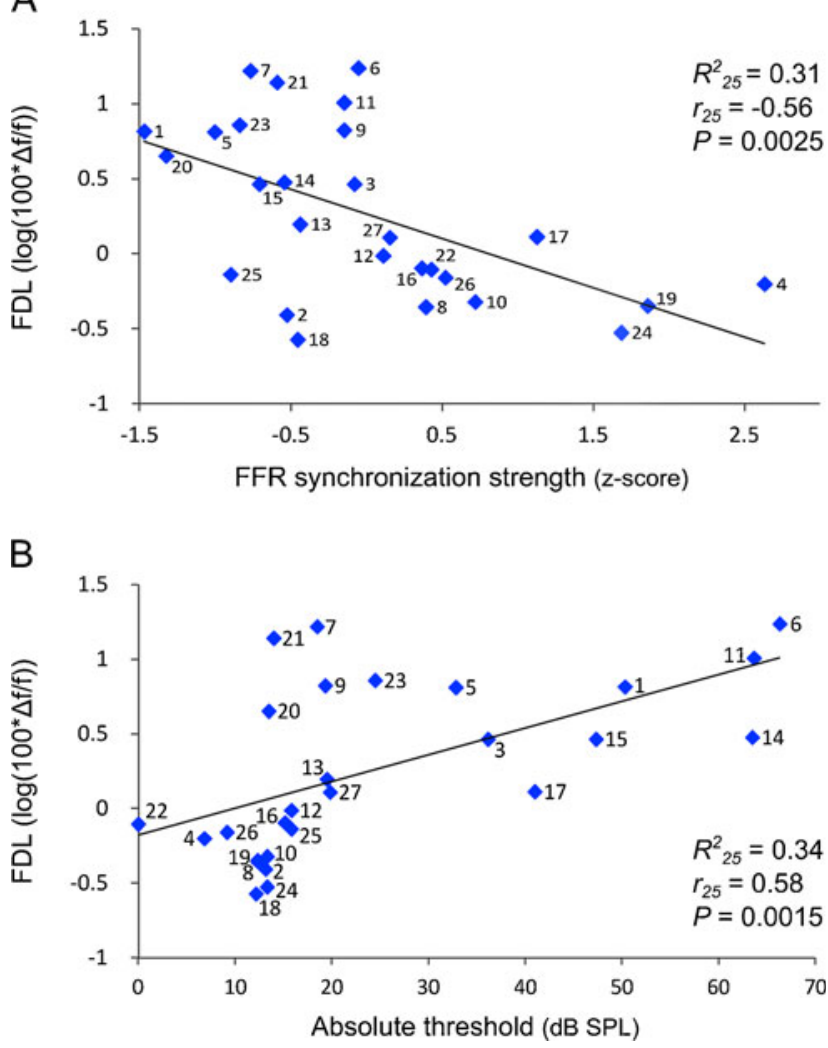

FIG. 1. Contributions of subcortical temporal coding and hearing loss to FDLs. Subcortical temporal coding is indexed by FFR synchronization strength and the degree of $\mathrm{CHL}$ is indexed by absolute thresholds. A Correlation between FFR synchronization strength and FDLs: this correlation held when both absolute thresholds and age were partialed out. B Correlation between absolute thresholds and FDLs: this correlation held when both FFR synchronization strength and age were partialed out. Data points for individual participants are numbered as in Table 1.

article to describe the statistical relationship between two variables.

Aging contributes to pure tone frequency discrimination via its influence on FFR synchronization strength

Age was also significantly correlated with FDLs $\left(r_{25}=0.39, P=0.043\right)$, but not when either the FFRs or absolute thresholds were partialed out (resp., $r_{24}=0.15$, $P=0.45$ and $r_{24}=0.30, P=0.14$ ). Hence, no evidence was found for an independent contribution of age on frequency discrimination. Age and absolute threshold were not correlated for the participants of this study $\left(r_{25}\right.$ $=0.28, P=0.16)$. However, age was significantly correlated with FFR synchronization strength $\left(r_{25}=-0.51\right.$, $P=0.0072)$ and group delay $\left(r_{23}=0.52, P=0.0079\right)$ (Fig. 2A and $\mathrm{B}$ ), and these correlations held when absolute thresholds were partialed out (correlation with
A

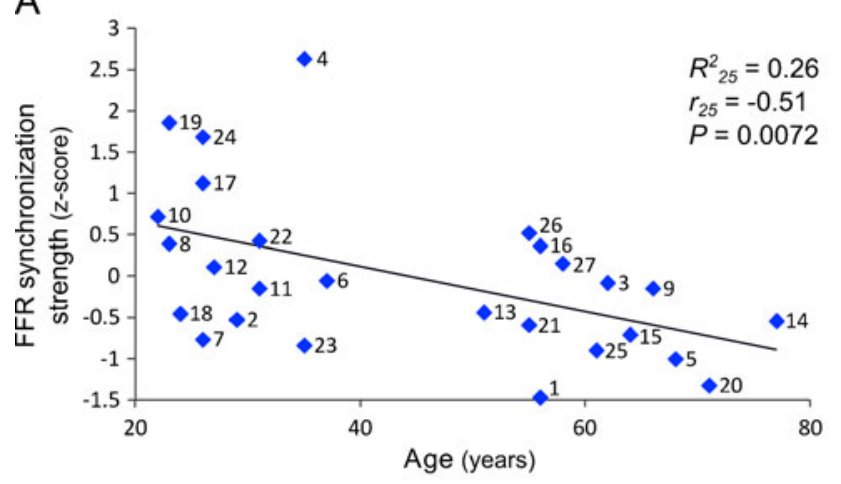

B

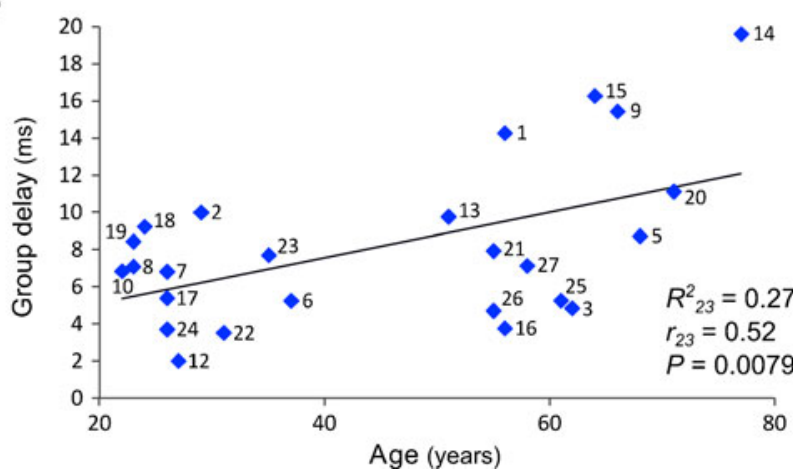

FIG. 2. Relations between age and subcortical temporal coding. The strength of phase locking is indexed by FFR scores and the latency of the subcortical response is indexed by its group delay. Age was correlated with FFR synchronization strength (A) and group delay (B), and the correlations held when absolute thresholds were partialed out. Data points for individual participants are numbered as in Table 1. Group delays could not be measured for participants 4 and 11 .

FFR synchronization strength: $r_{24}=-0.46, P=0.019$; with group delay: $r_{22}=0.42, P=0.040$ ). This suggests that the relation between age and pure tone frequency discrimination may be mediated by the detrimental effect of age on FFR synchronization strength.

Summary results: pure tone frequency discrimination is dependent on independent contributions of absolute thresholds and FFR synchronization strength

To clarify the respective contributions of FFR synchronization strength, absolute threshold, and age to frequency discrimination, a stepwise linear regression was performed using backward selection to determine the combination of FFR synchronization strength, absolute threshold, and age that best predicted performance in the discrimination task. Age did not make a significant contribution (standardized coefficient: $\beta=0.080, \mathrm{~T}_{24}=0.46, P=0.65$ ) and was removed from the regression. The best regression model was a combination of FFR synchronization strength 
and absolute threshold $\left(r^{2}\right.$ adj $=0.45, F_{2,24}=11.65$, $P=0.00029)$. Both regression coefficients were significant (standardized absolute threshold coefficient: $\beta=0.45, \mathrm{~T}_{24}=2.93, P=0.0074$; standardized FFR synchronization strength coefficient: $\beta=-0.42, \mathrm{~T}_{24}=2.70$, $P=0.012)$. The results of the regression analysis are plotted in Figure 3, a three-dimensional scatterplot where each point represents a participant's FFR synchronization strength, FDL, and absolute threshold value in the $x, y$, and $z$ dimensions respectively, and on which the best model is represented by a regression plane fitted to the points. Higher FFR synchronization strength and lower absolute thresholds are associated with lower (better) FDLs.

\section{DISCUSSION}

Contribution of subcortical neural synchrony to frequency discrimination

The present study shows a link between FFR synchronization strength and frequency discrimination in listeners with a wide range of ages and absolute thresholds: Behavioral frequency discrimination thresholds were reflected in the fidelity of frequency coding at the brainstem level. This finding is consistent with some previous studies relating behavioral frequency discrimination for complex tones to measures of periodicity strength in the FFR in normalhearing listeners (Bidelman et al. 2011; Krishnan et al. 2010, 2012; Smalt et al. 2012; Carcagno and Plack 2011), although one of these (Bidelman et al. 2011) did so for English-speaking musicians but not for English- speaking non-musicians nor Chinese-speaking nonmusicians. However, a previous study that investigated the relation between FFR responses and frequency discrimination for pure tones could not establish a link between them (Clinard et al. 2010).

The discrepancy among studies assessing the relation between FFR neural phase locking and behavioral frequency discrimination might be partly explained by the stimuli used and/or by the kind of listeners tested. First, whereas for pure tones listeners may benefit somewhat from place cues arising from the steep low-frequency side of the excitation pattern, this cue may be weaker for complex tones, whose discrimination may therefore rely more strongly on periodicity cues. Second, complex sounds of various pitch salience were used in Krishnan et al. (2010, 2012) and Smalt et al. (2012), and the relations between FFR neural phase locking and F0 discrimination observed are mediated by the consistent effects that manipulating pitch salience had on FFR measures and F0 discrimination. In other words, the correlations were driven by variability in the stimuli, rather than variability between listeners. No such stimulus manipulation was present in Clinard et al. (2010) or in the present study, which used pure tones. Third, the longterm pitch experience of the participants tested can influence the observation of a link between FFR measures and frequency discrimination: Musicians have both better pitch discrimination (Micheyl et al. 2006) and stronger FFR pitch strength (Parbery-Clark et al. 2009), and a recent study found a correlation between the FFR and discrimination of residue pitch in musicians but not in non-musicians (irrespective of their tone-language expertise) (Bidelman et al. 2011).

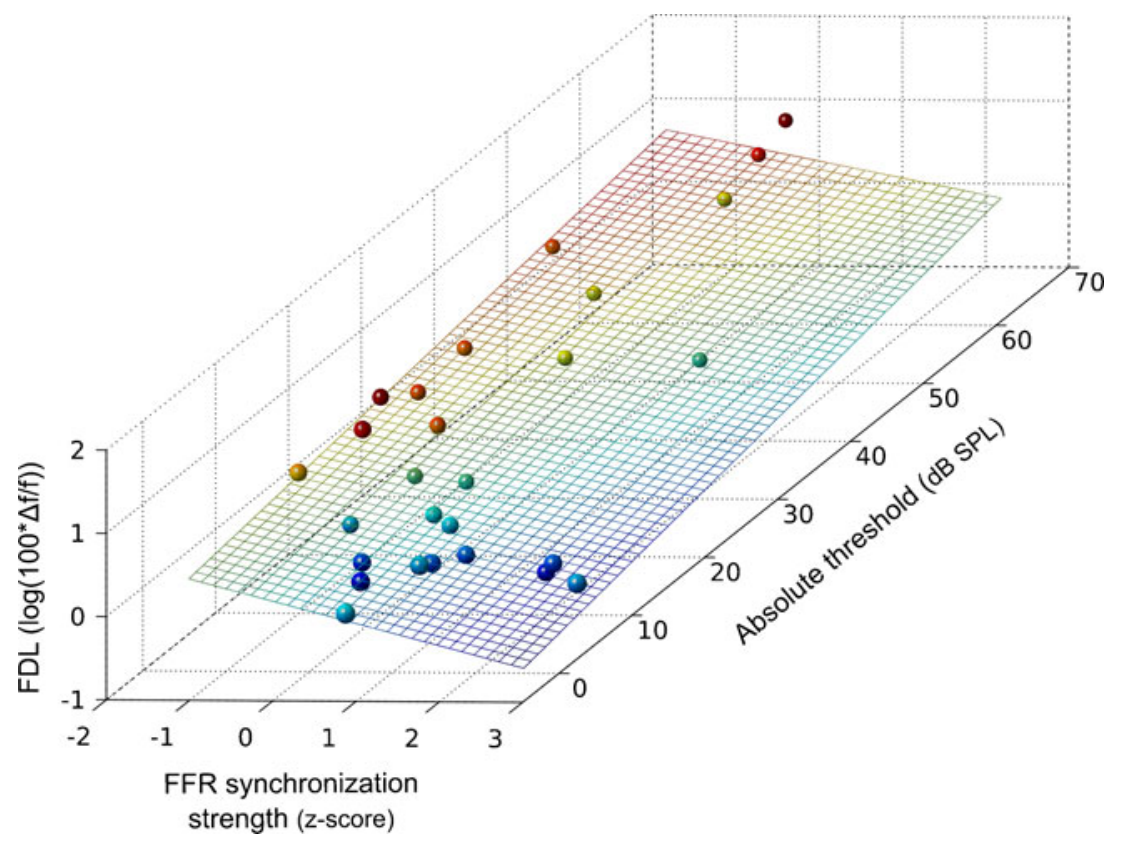

FIG. 3. Regression analysis: FDLs were best predicted by a linear combination of absolute threshold and composite FFR synchronization strength. Higher FFR synchronization strength and lower absolute thresholds were associated with lower (better) FDLs. Age and group delay did not make independent contributions to the model. Colors: The blue-to-red color map for the dots and the regression plane indicates better-to-worse FDLs. 
In the present study, ten of the 27 participants reported musical training (having played a musical instrument for at least 1 year, with an average of $11.8 \pm$ 14.4 years), so music expertise may have contributed to the correlation between FFR synchronization strength and FDLs. It cannot however fully account for the correlation, as it is still significant when only participants with no musical training are kept in the analysis $\left(n=17, r_{15}=-0.49, P=0.046\right)$.

Using pure tones, Clinard et al. (2010) did not observe a correlation between FFR measures and FDLs, contrary to the present study. One difference that could partly explain the different outcomes is that, in the present study, FFR synchronization strength was computed on a larger number of sweeps, as six conditions with similar frequencies (included in the study so as to calculate group delay) were averaged together. However, the zero-order correlation between FFR synchronization strength and FDL remains significant when only the FFR to the $660-\mathrm{Hz}$ condition is included $\left(r_{25}=-0.44\right.$, $P=0.022)$. Another possible explanation is that the present study tested both normal-hearing and hearingimpaired listeners whereas Clinard et al. (2010) focused on normal-hearing listeners. This may have resulted in more variability in FDLs or FFR synchronization strengths in the present study, increasing the data span of either the FDLs or the FFRs and making more likely the emergence of a correlation. However, the correlation remains significant when only participants with an absolute threshold better than $20 \mathrm{~dB}$ HL at $660 \mathrm{~Hz}$ are included ( $\left.n=19, r_{17}=-0.54, P=0.017\right)$.

The present study is unique in that it investigated concurrently the contributions of FFR synchronization strength, age, and absolute thresholds on FDLs (or pure tone discrimination) and used partial correlations and linear regression to weigh the respective contributions of these three factors. FFR synchronization strength was not the only factor accounting for frequency discrimination: absolute thresholds predicted FDLs independently of FFR measures. This suggests that additional mechanisms independent from the neural phase locking indexed by the FFR may contribute to frequency discrimination. First, the FFR-being a scalp-recorded response representing activity averaged over a large number of neurons-reflects a population-wide neuronal response, with some spike periodicities likely to be smeared out and not represented in the FFR. As such, additional neural phase locking not indexed by the FFR may play a role and FFR synchronization strength measures may underestimate the contribution of subcortical neural phase locking to frequency discrimination. Second, frequency codes not based on phase locking may play a complementary role. One such mechanism could be place-rate coding, as broader auditory filters are associated with higher absolute thresholds (Glasberg and Moore 1986). However, in that view, the similar weights in the regression model for the contributions of absolute thresholds and FFR synchronization strength contrast with previous reports of weak correlations between pure tone frequency discrimination and frequency resolution (Moore and Peters 1992; Tyler et al. 1983), and the subsequent inference that temporal coding may be dominant for low-frequency pure tones.

Effects of age and absolute thresholds on subcortical neural synchrony

An additional aim of the present study was to determine the effects of age and absolute thresholds on the subcortical neural synchrony indexed by the FFR. Age and absolute thresholds seemed to contribute to pure tone frequency discrimination performance by separate mechanisms, with age but not absolute threshold being related to FFR synchronization strength, and absolute threshold but not age making a contribution to frequency discrimination independently from FFR synchronization strength. Hence, biological processes related to age but not to absolute threshold seem to affect the subcortical neural synchrony indexed by the FFR. This result contrasts with a previous study by Plyler and Krishnan (2001) who reported poorer FFR neural synchrony in hearing-impaired listeners than in normal-hearing listeners. However, in their study the effect of age was not controlled (listeners ranged from 20 to 67 years).

The absence of a correlation between absolute threshold and FFR synchronization strength in the present study was unexpected in light of behavioral studies reporting an influence of hearing thresholds on measures of temporal coding such as detection of lowrate frequency modulation (Strelcyk and Dau 2009), interaural phase discrimination (Lacher-Fougère and Demany 2005; Hopkins and Moore 2011), and discrimination of unresolved "frequency-shifted" complex tones (Hopkins and Moore 2007, 2011). The absence of a correlation might be due to the moderate number of hearing-impaired participants in the present study. As the low-pass characteristics of brainstem phase locking limits the FFR to frequencies below $1.5 \mathrm{kHz}$ (Skoe and Kraus 2010), the present study used low frequencies for which audibility is usually more preserved with agerelated hearing impairment (Schmiedt 2010). Consequently, only eight participants out of 27 had an absolute threshold above $20 \mathrm{~dB} \mathrm{HL}$ at $660 \mathrm{~Hz}$. Another possibility is that CHL may degrade temporal cues not reflected in the FFR, such as across-channel timing differences (Oxenham et al. 2004; Cedolin and Delgutte 2010; but see Carlyon et al. 2012) or within-channel periodicities that may be smeared out due to the fact 
that the FFR is scalp-recorded and represents neural activity averaged over entire populations of neurons. Finally, it is also possible that many of the previous studies suggesting a link between CHL and temporal coding may have been confounded by the effects of age: Hearing-impaired participants were always older than normal-hearing participants, even if significant correlations between age and measures of temporal coding were not always found (Lacher-Fougère and Demany 2005, some conditions of Hopkins and Moore 2011).

The deterioration of subcortical neural synchrony, as measured by the FFR, with aging is consistent with previous studies using behavioral tasks (Hopkins and Moore 2011), auditory brainstem responses (Clinard et al. 2010; Vander Werff and Burns 2011; Konrad-Martin et al. 2012) and animal physiology (Walton 2010). In contrast, the disappearance of the correlation between age and frequency discrimination when absolute thresholds are controlled is not consistent with previous studies that showed an effect of age when the audiograms between younger and older participants were matched (He et al. 1998) or when all participants had thresholds within the normal range (Clinard et al. 2010). Explanations for this inconsistency might lie in residual differences between audiograms in the two aforementioned studies. Differences at $8 \mathrm{kHz}$ are visible in He et al. (1998, Fig. 1) and older participants in Clinard et al. (2010) might have had worse thresholds than young listeners even though they were all in the normal range. Another explanation could be a lack of power in the present study: For example, the lack of listeners of 'intermediate' age might weaken the correlation between age and frequency discrimination and make the correlation insignificant when the variance shared with absolute thresholds is held constant.

The disappearance of the correlation between age and frequency discrimination when FFR synchronization strength is controlled suggests, together with the finding that age did not make an independent contribution to the regression model, that the deteriorating effect of age on frequency discrimination is mediated via neural synchrony. Evidence from animal studies suggests that aging affects temporal processing at multiple levels in the auditory system (Canlon et al. 2010) and in particular leads to reduced neural firing synchrony. The present data suggesting a link between aging and subcortical neural synchrony are also consistent with previous findings showing that the effect of age on pitch discrimination is dependent on frequency, with the age effect being larger for lower frequencies (He et al. 1998), for which temporal mechanisms are thought to dominate pitch discrimination (Sek and Moore 1995). Temporal processing has also been proposed recently to mediate the agerelated deterioration of pitch processing in melodies (Russo et al. 2012).

\section{CONCLUSIONS}

The present findings suggest a link between subcortical neural synchrony and frequency discrimination that does not depend on age or absolute thresholds. An additional contribution of absolute thresholds to frequency discrimination is also evidenced. Overall, the results suggest that frequency discrimination for pure tones depends on independent contributions of the phase locking reflected in the FFR, and additional mechanisms degraded with CHL. These additional mechanisms may include phase locking not reflected in the FFR or place coding.

\section{ACKNOWLEDGMENTS}

This work was supported by MRC grant G0900591

\section{REFERENCES}

Anderson DJ, Rose JE, Hind JE, Brugge JF (1971) Temporal position of discharges in single auditory nerve fibers within the cycle of a sine-wave stimulus: frequency and intensity effects. J Acoust Soc Am 49:1131-1139

Anderson S, Parbery-Clark A, White-Schwoch T, Kraus N (2012) Aging affects neural precision of speech encoding. J Neurosci 32:14156-14164

Batra R, Kuwada S, Maher VL (1986) The frequency-following response to continuous tones in humans. Hear Res 21:167-177

Bidelman GM, Gandour JT, Krishnan A (2011) Musicians and tonelanguage speakers share enhanced brainstem encoding but not perceptual benefits for musical pitch. Brain Cogn 77:1-10

Canlon B, Illing RB, Walton J (2010) Cell biology and physiology of the aging central auditory pathway. In: Gordon-Salant S, Frisina RD, Popper AN, Fay RR (eds) The aging auditory system. Springer, New York, pp 39-72

Carcagno S, Plack CJ (2011) Subcortical plasticity following perceptual learning in a pitch discrimination task. J Assoc Res Otolaryngol 12:89-100

Carlyon RP, Long CJ, Micheyl G (2012) Across-channel timing differences as a potential code for the frequency of pure tones. J Assoc Res Otolaryngol 13:159-171

Cedolin L, Delgutte B (2010) Spatio-temporal representation of the pitch of harmonic complex tones in the auditory nerve. J Neurosci 30:12712-12724

Clinard CG, Tremblay KL, Krishnan AR (2010) Aging alters the perception and physiological representation of frequency: evidence from human frequency-following response recordings. Hear Res 264:48-55

CoHen MF (1982) Detection threshold microstructure and its effect on temporal integration data. J Acoust Soc Am 71:405-409

DE Cheveigné A (2005) Pitch perception models. In: Plack CJ, Oxenham A, Fay RR, Popper AN (eds) Pitch-neural coding and perception. Springer, New York, pp 169-233

Glasberg BR, Moore BC (1986) Auditory filter shapes in subjects with unilateral and bilateral cochlear impairments. J Acoust Soc Am 79:1020-1033 
Gockel He, Carlyon RP, Mehta A, Plack CJ (2011) The frequency following response (FFR) may reflect pitch-bearing information but is not a direct representation of pitch. J Assoc Res Otolaryngol 12:767-782

HARRISON RV, Evans EF (1979) Some aspects of temporal coding by single cochlear fibres from regions of cochlear hair cell degeneration in the guinea pig. Arch Otorhinolaryngol 224:71-78

He N, Dubno JR, Mills JH (1998) Frequency and intensity discrimination measured in a maximum-likelihood procedure from young and aged normal-hearing subjects. J Acoust Soc Am 103:553-565

Heinz MG, Colburn HS, Carney LH (2001) Evaluating auditory performance limits: I. One-parameter discrimination using a computational model for the auditory nerve. Neural Comput 13:2273-2316

Heinz MG, Swaminathan J, Boley JD, Kale S (2010) Across-fiber coding of temporal fine-structure: effects of noise-induced hearing loss on auditory-nerve responses. In: Lopez-Poveda EA, Palmer AR, Meddis R (eds) The neurophysiological bases of auditory perception. Springer, New York, pp 621-630

Hopkins K, Moore BCJ (2007) Moderate cochlear hearing loss leads to a reduced ability to use temporal fine structure information. J Acoust Soc Am 122:1055-1068

Hopkins K, Moore BCJ (2011) The effects of age and cochlear hearing loss on temporal fine structure sensitivity, frequency selectivity, and speech reception in noise. J Acoust Soc Am 130:334-349

Konrad-Martin D, Dille MF, McMillan G, Griest S, McDermott D, FAusti SA, Austin DF (2012) Age-related changes in the auditory brainstem response. J Am Acad Audiol 23:18-35

Krishnan A, Bidelman GM, Gandour JT (2010) Neural representation of pitch salience in the human brainstem revealed by psychophysical and electrophysiological indices. Hear Res 268:60-66

Krishnan A, Bidelman GM, Smalt CJ, Ananthakrishnan S, Gandour JT (2012) Relationship between brainstem, cortical and behavioral measures relevant to pitch salience in humans. Neuropsychologia 50:2849-2859

Lacher-Fougère S, Demany L (2005) Consequences of cochlear damage for the detection of interaural phase differences. J Acoust Soc Am 118:2519-2526

Lee KM, Skoe E, Kraus N, Ashley R (2009) Selective subcortical enhancement of musical intervals in musicians. J Neurosci 29:5832-5840

Marmel F, Parbery-Clark A, Skoe E, Nicol T, Kraus N (2011) Harmonic relationships influence auditory brainstem encoding of chords. Neuroreport 22:504-508

Micheyl C, Delhommeau K, Perrot X, Oxenham AJ (2006) Influence of musical and psychoacoustical training on pitch discrimination. Hear Res 219:36-47

Micheyl C, Moore BJC, Carlyon RP (1998) The role of excitationpattern cues and temporal cues in the frequency and modulation-rate discrimination of amplitude-modulated tones. J Acoust Soc Am 104:1039-1050

Miller RL, Schilling JR, Franck KR, Young ED (1997) Effects of acoustic trauma on the representation of the vowel $/ \varepsilon /$ in cat auditory nerve fibers. J Acoust Soc Am 101:3602-3616

Moore BCJ (1973A) Frequency difference limens for narrow bands of noise. J Acoust Soc Am 54:888-896

MoORE BCJ (1973B) Frequency difference limens for short-duration tones. J Acoust Soc Am 54:610-619

Moore BCJ, Carlyon RP (2005) Pitch for hearing-impaired listeners and cochlear implantees. In: Plack CJ, Oxenham A, Fay RR, Popper AN (eds) Pitch-neural coding and perception. Springer, New York, pp 234-277

Moore BCJ, Peters RW (1992) Pitch discrimination and phase sensitivity in young and elderly subjects and its relationship to frequency selectivity. J Acoust Soc Am 91:2881-2893
Moore BCJ, Huss M, Vickers DA, Glasberg BR, Alcántara JI (2000) A test for the diagnosis of dead regions in the cochlea. Br J Audiol 34:205-224

Moushegian G, Rupert AL, Stillman RD (1973) Scalp-recorded early responses in man to frequencies in the speech range. Electroen Clin Neuro 35:665-667

Oxenham AJ, Bernstein JGW, Penagos H (2004) Correct tonotopic representation is necessary for complex pitch perception. Proc Natl Acad Sci U S A 101:1421-1425

Parbery-Clark A, Anderson S, Hittner E, Kraus N (2012) Musical experience offsets age-related delays in neural timing. Neurobiol Aging 33:1483.e1-1483.e4

Parbery-Clark A, Skoe E, Kraus N (2009) Musical experience limits the degradative effects of background noise on the neural processing of sound. J Neurosci 29:14100-14107

Plack CJ (2005) The sense of hearing. Lawrence Erlbaum, London

Plyler PN, Krishnan AK (2001) Human frequency-following responses: representation of second formant transitions in normalhearing and hearing-impaired listeners. J Am Acad Audiol 12:523-533

Russo NM, Skoe E, Trommer B, Nicol T, Zecker S, Bradlow A, Kraus N (2008) Deficient brainstem encoding of pitch in children with autism spectrum disorders. Clin Neurophysiol 119:1720-1731

Russo N, Nicol T, Trommer B, Zecker S, Kraus N (2009) Brainstem transcription of speech is disrupted in children with autism spectrum disorders. Developmental Sci 12:557-567

Russo FA, Ives DT, Goy H, Pichora-Fuller MK, Patterson RD (2012) Age-related difference in melodic pitch perception is probably mediated by temporal processing: empirical and computational evidence. Ear Hear 33:177-186

Sek A, Moore BCJ (1995) Frequency discrimination as a function of frequency, measured in several ways. J Acoust Soc Am 97:24792486

Schmiedt RA (2010) The physiology of cochlear presbycusis. In: Gordon-Salant S, Frisina RD, Popper AN, Fay RR (eds) The aging auditory system. Springer, New York, pp 9-38

Skoe E, Kraus N (2010) Auditory brainstem response to complex sounds: a tutorial. Ear Hear 31:302-324

Smalt CJ, Ananthanarya K, Bidelman GM, Ananthakrishnan S, Gandour J (2012) Distortion products and their influence on representation of pitch-relevant information in the human brainstem for unresolved harmonic complex tones. Hear Res 292:26-34

Song JH, Skoe E, Banai K, Kraus N (2012) Training to improve hearing speech in noise: biological mechanisms. Cereb Cortex 22:1180-1190

Stillman RD, Crow G, Moushegian G (1978) Components of the frequency-following potential in man. Electroen Clin Neuro 44:438-446

Strelcyk O, DAU T (2009) Relations between frequency selectivity, temporal fine-structure processing, and speech reception in impaired hearing. J Acoust Soc Am 125:3328-3345

Strouse A, Ashmead DH, Ohde RN, Grantham DW (1998) Temporal processing in the aging auditory system. J Acoust Soc Am 104:2385-2399

Tyler RS, Wood EJ, Fernandes M (1983) Frequency resolution and discrimination of constant and dynamic tones in normal and hearing-impaired listeners. J Acoust Soc Am 74:1190-1199

VANDER WERFF KR, Burns KS (2011) Brain stem responses to speech in younger and older adults. Ear Hear 32:168-180

WALTON JP (2010) Timing is everything: temporal processing deficits in the aged auditory brainstem. Hear Res 264:63-69

Woolf NK, Ryan AF, Bone RC (1981) Neural phase-locking properties in the absence of cochlear outer hair cells. Hear Res 4:335-346

Worden FG, Marsh JT (1968) Frequency-following (microphoniclike) neural responses evoked by sound. Electroen Clin Neuro 25:42-52 